\title{
Erratum to: Expression analysis of loci associated with type 2 diabetes in human tissues
}

\author{
C. Cotsapas • L. Prokunina-Olsson • C. Welch • \\ R. Saxena $\cdot$ C. Weaver $\cdot$ N. Usher $\cdot$ C. Guiducci • \\ S. Bonakdar $\cdot$ N. Turner $\cdot$ B. LaCroix • J. L. Hall
}

Published online: 16 October 2010

(C) Springer-Verlag 2010

\section{Erratum to: Diabetologia}

DOI 10.1007/s00125-010-1861-2

Unfortunately, in Fig. 2, the legend did not match the figure panels. The legend should have read:

Fig. 2 Immunohistochemical localisation of HHEX. Moderate to strong immunoreactivity was found in pancreatic acini and islets (c). Localisation was nuclear and cytoplasmic. Pancreatic ducts (b) exhibited moderate, exclusively nuclear staining. Liver parenchyma (a) and colonic epithelia (d) showed little immunoreactivity or staining

The online version of the original article can be found at http://dx.doi. org/10.1007/s00125-010-1861-2.

\footnotetext{
C. Cotsapas · R. Saxena · C. Guiducci · S. Bonakdar

Broad Institute,

Cambridge, MA, USA

L. Prokunina-Olsson

Laboratory of Translational Genomics, Division of Cancer

Epidemiology and Genetics, National Cancer Institute, NIH,

Bethesda, MD, USA

C. Welch $\cdot$ C. Weaver $\cdot$ N. Usher $\cdot$ N. Turner $\cdot$ B. LaCroix $\cdot$

J. L. Hall ( $\square)$

Department of Medicine, Lillehei Heart Institute,

4-280 NHH, 312 Church St SE,

Minneapolis, MN 55455, USA

e-mail: jlhall@umn.edu

C. Cotsapas $\cdot$ R. Saxena

Department of Medicine, Harvard Medical School,

Boston, MA, USA
}

J. L. Hall

Developmental Biology Center, University of Minnesota, Minneapolis, MN, USA

C. Cotsapas $\cdot$ R. Saxena

Center for Human Genetic Research, Department of Medicine, Massachusetts General Hospital,

Boston, MA, USA

R. Saxena

Department of Anesthesia, Critical Care and Pain Medicine, Massachusetts General Hospital,

Boston, MA, USA 